\title{
The magnetic field of the evolved star W43A
}

\author{
Nikta Amiri, ${ }^{a, c}$ Wouter Vlemmings ${ }^{b}$ and Huib Jan van Langevelde ${ }^{a, c}$ \\ ${ }^{a}$ Joint Institute for VLBI in Europe (JIVE) \\ The Netherlands \\ ${ }^{b}$ Argelander Institute for Astronomy, University of Bonn \\ Germany \\ ${ }^{c}$ Leiden Observatory, Leiden University \\ The Netherlands \\ E-mail: amiri@jive.nl, wouter@astro.uni-bonn.de, langevelde@jive.nl
}

Planetary nebulae (PNe) often show large departures from spherical symmetry. The origin and development of these asymmetries is not clearly understood. The most striking structures are the highly collimated jets that are already observed in a number of evolved stars before they enter the PN phase. The aim of this project is to observe the Zeeman splitting of the $\mathrm{OH}$ maser of the W43A star and determine the magnetic field strength in the low density region. The $1612 \mathrm{MHz}$ $\mathrm{OH}$ masers of W43A were observed with MERLIN to measure the circular polarization due to the Zeeman splitting of $1612 \mathrm{OH}$ masers in the envelope of the evolved star W43A. We measured the circular polarization of the strongest $1612 \mathrm{OH}$ masers of W43A and found a magnetic field strength of $\sim 100 \mu G$. The magnetic field measured at the location of W43A OH masers confirms that a large scale magnetic field is present in $\mathrm{W} 43 \mathrm{~A}$, which likely plays a role in collimating the jet.

The 9th European VLBI Network Symposium on The role of VLBI in the Golden Age for Radio Astronomy and EVN Users Meeting

September 23-26, 2008

Bologna, Italy 


\section{Introduction}

Planetary nebulae (PNe), supposedly formed out of the ejected outer envelopes of AGB stars, often show large departures from spherical symmetry. The origin and development of these asymmetries is not clearly understood. The most striking structures are the highly collimated jets that are already observed in a number of evolved stars before they enter the PN phase. However, the origin of the collimation of the jet is still puzzling. Theoretical models have shown that collimated jets can be caused by magnetic field in evolved stars [1]. Studying the large scale magnetic field through polarization observations of different maser species in the circumstellar envelope (CSE) of these stars provides a unique tool to understand the role of the magnetic field in the process of jet collimation. The aim of this project is to observe Zeeman splitting of the $\mathrm{OH}$ maser of W43A and determine the magnetic field strength in the low density region. $\mathrm{H}_{2} \mathrm{O}$ maser polarization observations have revealed that the jet of $\mathrm{W} 43 \mathrm{~A}$ is likely magnetically collimated [3]. $\mathrm{OH}$ masers arising from the material surrounding the jet are predicted to also have a detectable magnetic field and our observation will thus be able to confirm the role of the magnetic field in collimating the jet in proto-PNe.

The $1612 \mathrm{MHz} \mathrm{OH}$ masers of W43A were observed with MERLIN in June 2007. The observations were done with maximum possible 256 spectral resolution since all 4 polarization channels were required. The source $3 \mathrm{C} 84$ was used for bandpass and polarization calibration. The resulting noise in the emission free channel was $65 \mathrm{mJy} /$ Beam.

\section{Results}

From the image plane we have obtained the typical double peak spectrum of the $\mathrm{OH}$ maser region of W43A. Figure 1 shows the velocity profile of the integrated flux of each channel in the I, V and P (polarization intensity) data cubes. The velocity spectrum of the total intensity data cube is a typical double peak profile with velocity in the range $27-43 \mathrm{Km} / \mathrm{s}$; despite the fact that W43A is a water fountain source and has much higher velocities in the $\mathrm{H}_{2} \mathrm{O}$ maser region (in the range -53 to $126 \mathrm{~km} / \mathrm{s}$ ). The brightest peak is red-shifted and the blue-shifted peak has a much lower brightness; only $3 \%$ of the red-shifted brightness. The peaks in the polarization intensity and circular polarization spectra are $10 \%$ linearly and $12 \%$ circularly polarized. The relatively low level of linear polarization excludes most non-Zeeman interpretations of the circular polarization [2].

Figure 2 shows the spatial distribution of the $\mathrm{OH}$ maser features of W43A together with $\mathrm{H}_{2} \mathrm{O}$ maser positions [3]. The offset positions are with respect to the reference feature. $\mathrm{H}_{2} \mathrm{O}$ maser features are indicated by filled circles. $\mathrm{OH}$ maser features are shown as triangles. Red and blue colors show the red-shifted and blue-shifted features. This map clearly shows that $\mathrm{H}_{2} \mathrm{O}$ maser occurs at the tips of a jet and the $\mathrm{OH}$ maser has a projected distance much closer to the star.

The actual measurement of the magnetic field strength may depend on a number of conditions, but is estimated to be $100 \mu G$. This value is consistent with the expected magnetic field strength of $70 \mu \mathrm{G}$ extrapolated from $\mathrm{H}_{2} \mathrm{O}$ maser observations [3]. A lower $\mathrm{OH}$ magnetic field is expected compared to $\mathrm{H}_{2} \mathrm{O}(85 \pm 33 \mathrm{mG})$ due to the higher density and shocked nature of the $\mathrm{H}_{2} \mathrm{O}$ masers at the tips of the jet. 


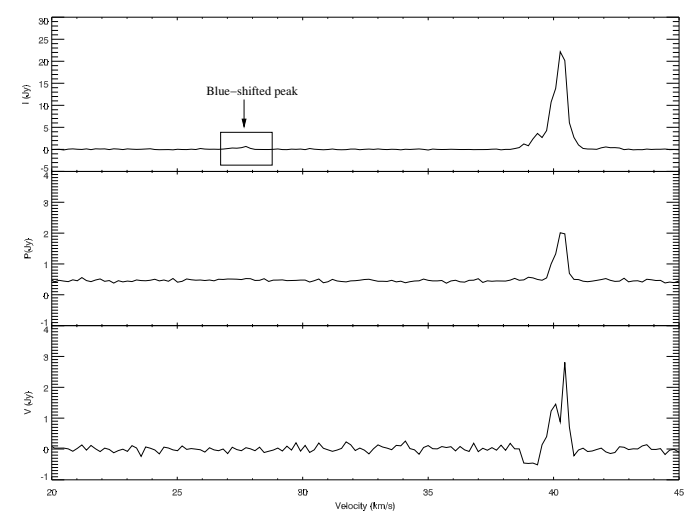

Figure 1: The $1612 \mathrm{MHz}$ spectra of W43A.

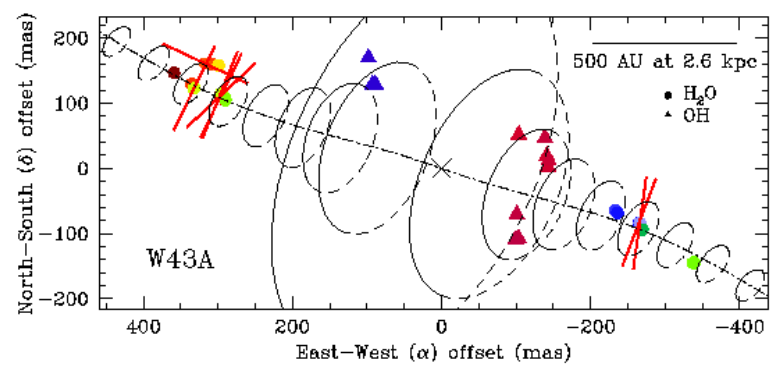

Figure 2: The spatial distribution of the $\mathrm{OH}$ and $\mathrm{H}_{2} \mathrm{O}$ maser features of W43A. $\mathrm{OH}$ maser positions are overlaid on $\mathrm{H}_{2} \mathrm{O}$ maser features [3].

\section{Conclusions}

The magnetic field and jet characteristics of W43A have previously been reported from $\mathrm{H}_{2} \mathrm{O}$ maser polarization observations in relation to the formation of non-spherical planetary nebulae. Here we present the detection of the magnetic field of $\sim 100 \mu G$ in the $\mathrm{OH}$ maser region surrounding the collimated jet. This confirms that a large scale magnetic field is present in W43A, which likely plays a role in collimating the jet.

\section{References}

[1] Blackman, E. G. \& Frank, A.\& Markiel, J. A.\& Thomas, J.H. \& Van Horn, H. M., 2001, Nature 409, 485

[2] Fish, V. L. \& Reid, M. J. 2006,ApJ, 164, 99

[3] Vlemmings, W. H. T. \& Diamond, P. J. \& Imai, H., 2006, Nature, 440, 58 